\title{
Математическое моделирование формирования аномальных напряжений горизонтального сжатия за счёт денудационных процессов
}

\author{
Мягков Д.С. \\ ИФ3 РАН, Москва,dsm@ifz.ru
}

\begin{abstract}
Аннотация. В работе рассматривается вопрос о физическом механизме, ответственном за формирование аномальных напряжений горизонтального сжатия в верхней части Земной коры, в том числе на Балтийском щите. В качестве такого механизма может выступать переход вещества верхней части Земной коры из закритического состояния за счёт денудации. Исследование ведётся методом численного моделирования. Представлены различные модели, в которых аномальные напряжения горизонтального сжатия формируются за счёт той или иной структуры экзогенных процессов (например - речной эрозии). Показаны форма и частей областей коры моделей, в которых за счёт денудации формируется значительный уровень аномальных напряжений горизонтального сжатия. Даны количественные связи уровня данных напряжений с амплитудами задаваемой в моделях денудации.
\end{abstract}

Ключевые слова: денудация, геодинамика, математическое моделирование, геомеханика.

\section{Mathematical modeling of the abnormal horizontal compression stresses formation due to denudation processes}

\author{
Myagkov D.S. \\ IPERAS, Moscow,dsm@ifz.ru
}

\begin{abstract}
The article reviews the issue of the physical mechanism responsible for the formation of anomalous stresses of horizontal compression in the upper part of the Earth's crust, including the Baltic Shield. Such a mechanism can be the transition of a substance from the upper part of the Earth's crust from a supercritical state due to denudation. The study is conducted by the method of numerical simulation. Various models are presented in which anomalous stresses of horizontal compression are formed due to one or another structure of exogenous processes (for example, river erosion). The shape and parts of the regions of the cortex of models are shown, in which a significant level of anomalous stresses of horizontal compression is formed due to denudation. Quantitative relationships of the level of these voltages with amplitudes specified in denudation models are given.
\end{abstract}

Key words: denudation, geodynamics, mathematical modeling, geomechanics.

\section{Введение}

В данной работе рассматривается вопрос о происхождении избыточных напряжений горизонтального сжатия, объяснение наличия которых в Земной коре является важной проблемой тектоники. Этот вопрос активно обсуждается с 50-х годов прошлого столетия и, до сих пор, не имеет однозначного решения. Рассматриваемая проблема имеет прикладное значение, т.к. аномальные напряжения горизонтального сжатия нередко создают затруднения при добыче полезных ископаемых и являются одной из серьёзнейших проблем при разработке рудных месторождений (Ребецкий и др., 2017), в том числе на российской территории (Кольский полуостров). В верхней части коры нормальный уровень горизонтальных напряжений соответствует формирующемуся при гравитационном сжатии упругой среды (к примеру, для коэффициента Пуассона 0.25 вертикальные нормальные напряжения превышаю горизонтальные в три раза). В коре многих платформ наблюдается иное соотношение вертикальных и горизонтальных напряжений, иногда даже последние в несколько раз превышают первые. Неоднократно предпринимались попытки объяснения данного явления с позиций тектоники литосферных плит и предлагались различные тектонические модели, для Балтийского щита преимущественно связывавшие формирование аномальных напряжений с давлением со стороны оси спрединга, не увенчавшиеся, в конечном итоге, успехом. Позднее были предложены другие модели, более согласованные с тектонофизическими данными о структуре напряжённого 
состояния Земной коры соответствующих регионов, в которых рассматриваемые напряжения горизонтального сжатия формировались за счёт денудации горных пород (Ребецкий, 2008).

\section{Денудационный механизм формирования аномальных напряжений горизонтального сжатия}

Опишем кратко предлагаемый механизм формирования аномальных напряжений горизонтального сжатия (подробнее см. Ребецкий, 2008). Верхние области коры находится, согласно результатам геомеханических исследований, в чисто упругом напряжённом состоянии. Отношение $\sigma_{x x}$ напряжений к $\sigma_{z z}$ равно v/(1-v), где v - коэффициент Пуассона. С глубиной давление, связанное с гравитационным напряжённым состоянием, достигает уровня, при котором среда переходит в закритическое состояние. Согласно Николаевскому (Николаевский, 1983) уровень напряжений, при котором развивается истинная (дислокационная) пластичность достигается на глубине Мохо, выше же в среде накапливаются разномасштабные разнонаправленные разрывные нарушения и деформация среды реализуется за счёт подвижек по данным нарушениям, причём подобное движение до-

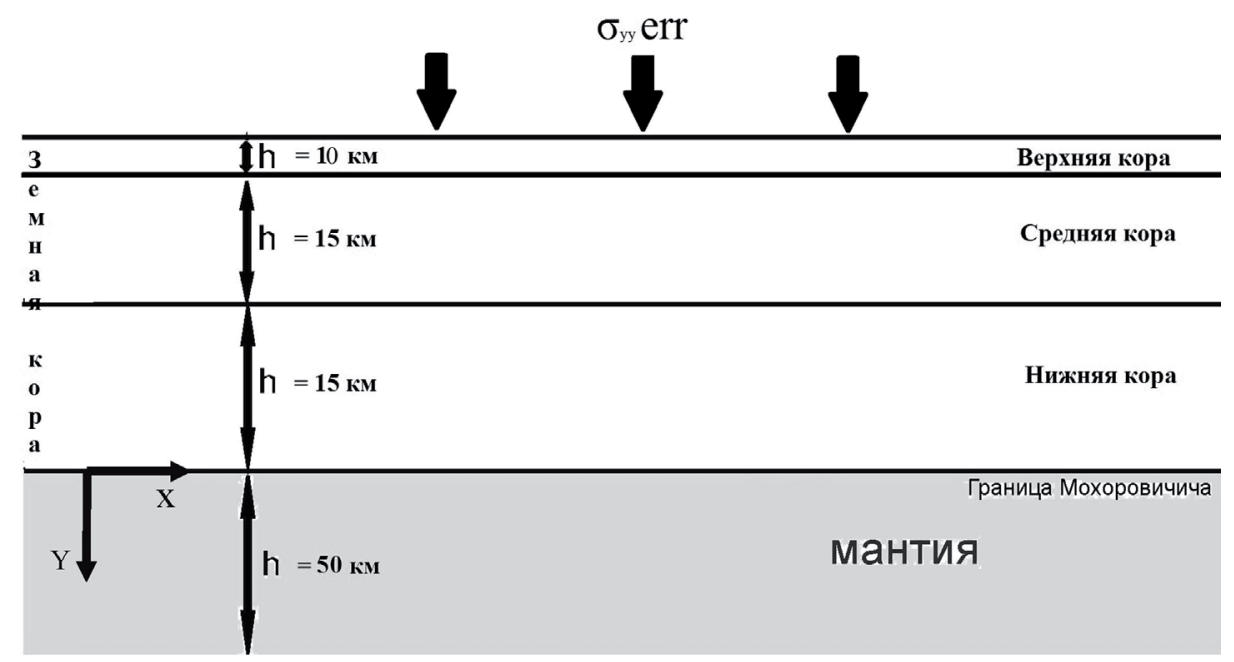

Рис. 1. Схема модели I. Fig. 1. Scheme of model I.

статочно схоже с пластическим течением и названо катакластическим. Таким образом, на некоторой глубине, которая в зависимости от состава, флюидонасыщенности и других параметров среды определяется примерно равной 2 и более км, за счёт катакластических процессов начинают выравниваться $\sigma_{x x}$ и $\sigma_{z z}$ напряжения, по мере роста давления приближаясь к отношению $1 / 1$ вместо v/(1-v). Если, далее, рассматриваемый участок среды окажется в области низких давлений (т.е. переместится выше глубины развития катакластических деформаций), разгрузка произойдёт по упругому закону, что, в условиях горизонтального стеснения вещества коры, приведёт к формированию остаточных напряжений горизонтального сжатия (точная аналитическая формула величины последних приведена в (Ребецкий, 2008)). Таким образом, денудационно-аккумулятивные процессы могут привести к формированию искомых аномальных напряжений в Верхней коре, если, конечно, амплитуда денудации достигает определённого уровня.

\section{Методика численного моделирования. Схемы моделей и характер нагружения}

Опишем кратко методику исследования. Исследование ведётся методом численного моделирования. Созданы несколько моделей участков континентальной литосферы и один - субдукционной зоны, в которых вводятся денудационные (и, если требуется, аккумулятивные) процессы требуемой структуры. Для численного расчёта используется конечно-разностная схема по методике Ю.П. Стефанова (Стефанов, 2005), которая представляет собой модифицированную для применения в задачах геомеханики схему Уилкинса (Уилкинс, 1967). В рамках данного подхода тела модели являются упруго-пластическими, причём используется специально адаптированных для геосре- 
ды неассоциированный закон пластического течения Друккера-Прагера-Николаевского. Все модели - двумерные. Тип напряжённого состояния - плоская деформация.

В работе представлены 4 различных модели. Модель I - модель континентальной литосферы без наличия рельефа основных границ (условно - модель платформы).Такая модель более остальных представленных соответствует коре Кольского полуострова. Схема модели представлена на рис. 1. Денудация вводится следующим образом: на первом этапе рассчитывается нормальное гравитационное напряжённое состояние. Далее на дневной поверхности задаются дополнительные $\sigma_{z z}$ напряжения, амплитуда которых соответствует мощности денудированного слоя (берётся 0.5-1.5 км). Наконец, на последнем этапе, напряжения снимаются, после чего формируются искомые аномальные напряжения в верхней части модели. Модель II схожа по строению с первой, однако в ней дополнительно задаётся уступ (стенка) на дневной поверхности амплитудой 2-4 км. Эта модель условно соответствует восточному флангу плоскогорья Тибет. Денудация в области плоскогорья задаётся аналогично, в опущенной части могут вводится аккумулятивные процессы (или не вводятся, в случае, если считается что реками удалена большая часть осадков). Третья модель представляет собой также участок континентальной литосферы, где денудация связана с формированием долины крупной реки (условно - одной из рек Тибетского нагорья). Модель IV представляет собой Японскую зону субдукции региона Северное Хонсю (подробно описана в работе (Ребецкий и др., 2018)).

\section{Результаты моделирования, выводы}

Проведённая серия численных экспериментов показала пригодность рассматриваемого физического механизма для объяснения формирования аномальных напряжений горизонтального сжатия и позволила установить некоторые закономерности данного процесса. Для формирования значимых по мощности областей превышения горизонтальных нормальных напряжений над вертикальными требовалась амплитуда денудации от 0.5 км и более. При этом формировались аномальные напряжения по амплитуде равные первым десяткам МПа (точное значение в зависимости от параметров нагружения и прочностных свойств коры модели). При амплитуде денудации от 1 км в модели I формировался слой мощностью более 3 км, где $\sigma_{x x}$ напряжения превышали вертикальные. На рисунке 2 представлена структура формирующегося напряжённого состояния в модели I, а именно - отношение вертикальных и горизонтальных нормальных напряжений (этот же параметр на рисунках 3 и 4 для моделей II и IV), при переходе среды в закритическое состояние этот параметр, как указывалось выше, постепенно принимает значение 1. Если он меньше единицы, мы имеем искомое аномальное состояние с сильным превышением уровня напряжений горизонтального сжатия над

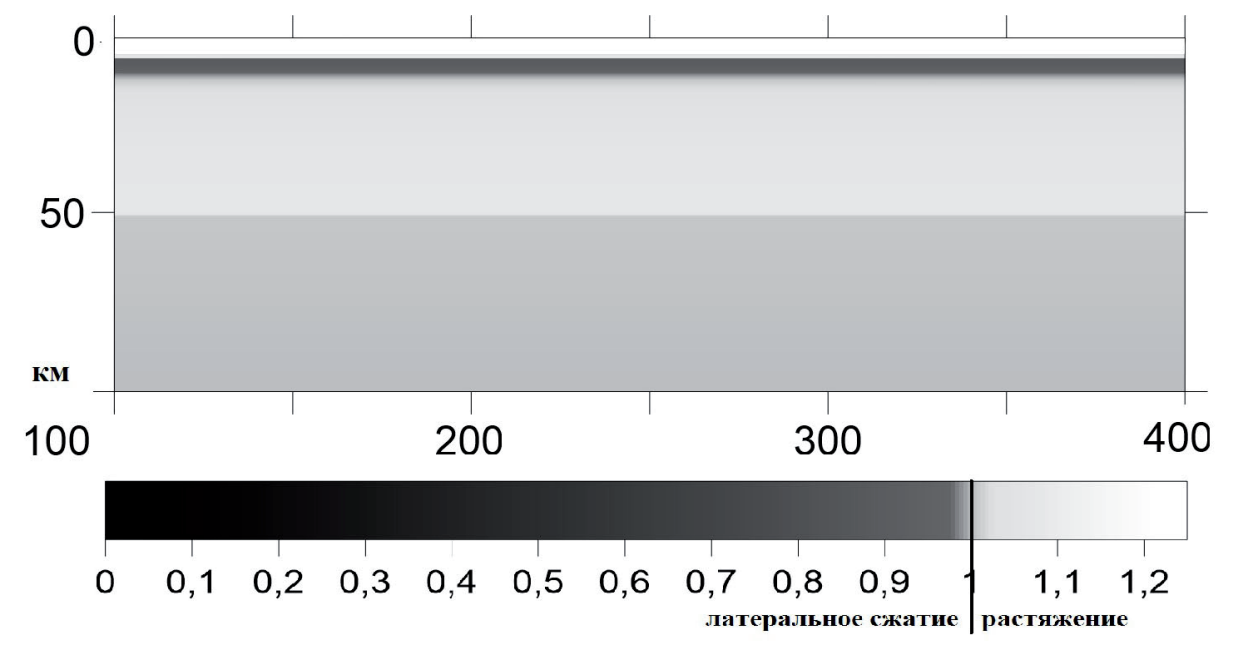

Рис. 2. Результаты моделирования формирования аномальных $\sigma_{x x}$ напряжений за счёт эрозии в модели I. На рисунке - параметр $\sigma_{y y} / \sigma_{x x}$, характеризующий тип напряжённого состояния.

Fig. 2. Results of modeling the formation of anomalous $\sigma_{x x}$ stresses due to erosion in model I. In the figure, the parameter $\sigma_{y y} / \sigma_{x x}$, characterizes the type of stress state. 


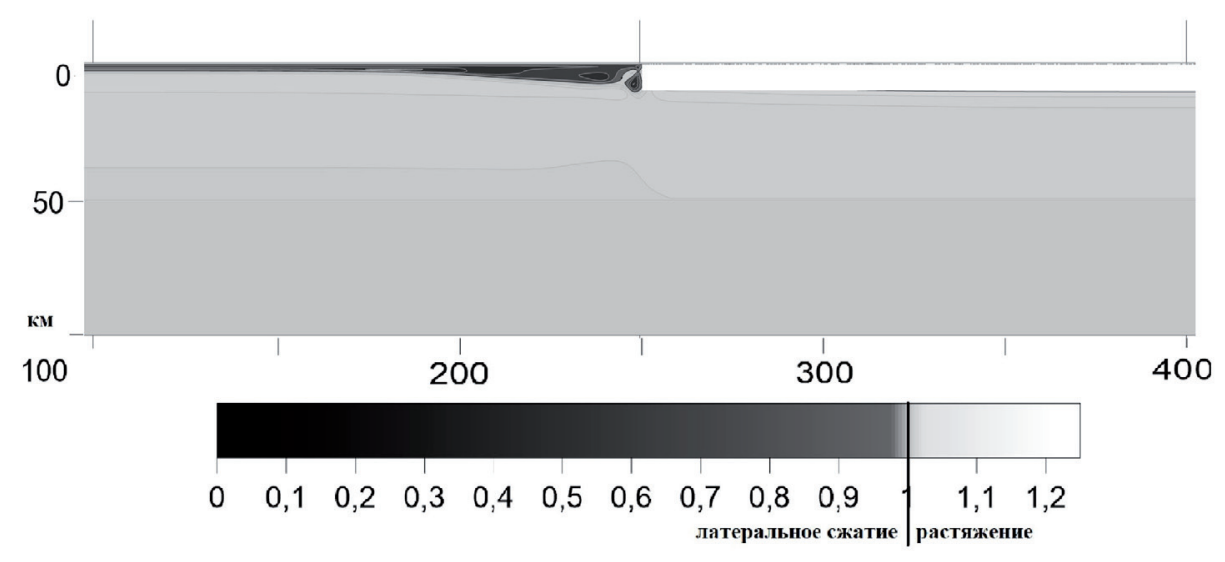

Рис. 3. Результаты моделирования формирования аномальных $\sigma_{x x}$ напряжений за счёт эрозии в модели II. На рисунке - параметр $\sigma_{y y} / \sigma_{x x}$, характеризующий тип напряжённого состояния.

Fig. 3. Results of modeling the formation of anomalous $\sigma_{x x}$ stresses due to erosion in model II. In the figure, the parameter $\sigma_{y y} / \sigma_{x x}$, characterizes the type of stress state.

гравитационным. В зависимости от структуры денудационных (либо ещё и аккумулятивных) процессов данный слой может иметь сложную геометрию (см. рисунок 3 для модели II с сильным перепадом рельефа), терять сплошность (см. рис. 4 для зоны субдукции Тохоку) или быть локализован (как для модели III с речной эрозией). Фактически, комбинацией рассмотренных полей напряжений можно объяснить достаточно сложные, с тектонофизической точки зрения, картины распределения напряжений в среде, учитывая комплексность экзогенного эрозионно-аккумулятивного процесса как пространственно, так и во времени. Важным преимуществом рассмотренного механизма является «уход» от необходимости объяснять формирование аномальных $\sigma_{x x}$ напряжений давлением со стороны оси спрединга, так как подобные модели, как говорилось выше, не соответствуют тектонофизическим данным.
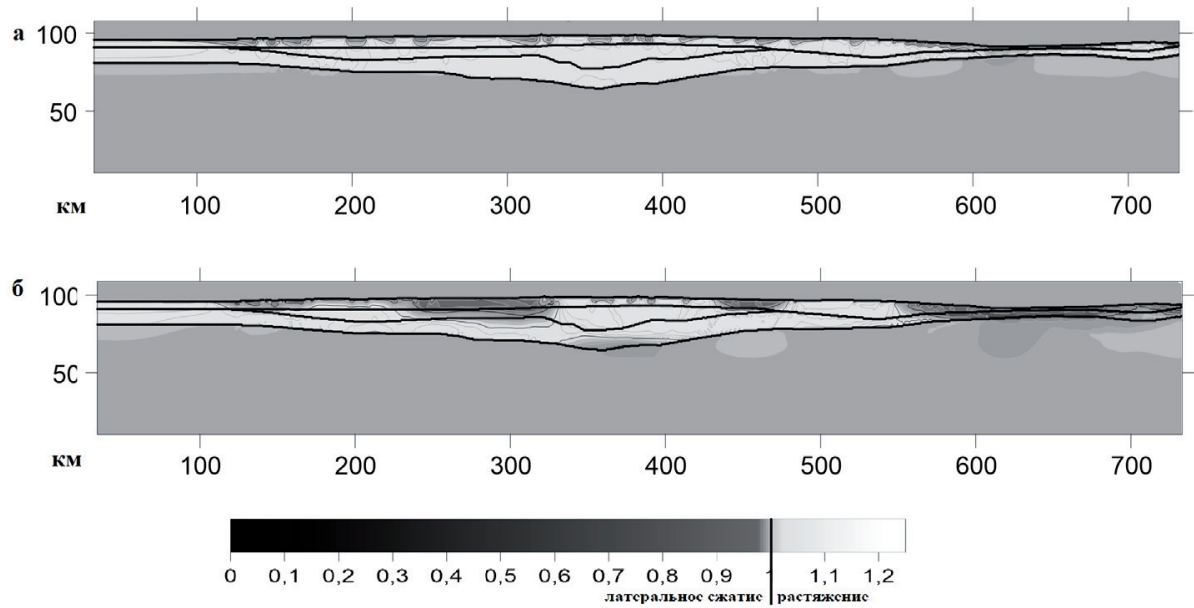

Рис. 4. Результаты моделирования формирования аномальных $\sigma_{x x}$ напряжений за счёт эрозии в модели IV (рис. б). На рисунке а приведено гравитационное напряжённое состояние до эрозионного воздействия. На рисунке - параметр $\sigma_{y y} / \sigma_{x x}$, характеризующий тип напряжённого состояния. Данные опубликованы в работе (Ребецкий и др., 2018).

Fig. 4. Results of modeling the formation of anomalous $\sigma_{x x}$ stresses due to erosion in model IV. Figure a shows the gravitational stress state before erosion. In the figure, the parameter $\sigma_{y y} / \sigma_{x x}$, characterizes the type of stress state.

Исследования выполнены при поддержке гранта РФФИ 18-35-00482 и Госзадания ИФЗ РАН. 


\section{Литература}

1. Николаевский В.Н. Механические свойства грунтов и теория пластичности // Механика твёрдых деформируемых тел. Т. 6. Итоги науки и техники. М.: ВИНИТИ АН СССР 1972. С. 5-81.

2. Ребецкий Ю.Л. О возможном механизме генерации в земной коре горизонтальных сжимающих напряжений // ДАН. 2008. Т. 423. № 4. С. 538-542.

3. Ребецкий Ю.Л., Погорелов В.В., Мягков Д.С., Ермаков В.А. О генезисе напряжений в коре островной дуги по результатам численного моделирования // Вестник КРАУНЦ. 2018. № 3. С. 54-73.

4. Ребецкий Ю.Л, Сим Л.А., Козырев А.А. О возможном механизме генерации избыточного горизонтального сжатия рудных узлов Кольского полуострова (Хибины, Ловозеро, Ковдор) // Геология рудных месторождений. 2017. Т. 59. № 4. С. 263-280.

5. Стефанов Ю.П. Некоторые особенности численного моделирования поведения упруго-хрупкопластичных материалов // Физ. мезомех. 2005. Т. 8. № 3. С. 129-142.

6. Уилкинс М.Л. Расчёт упруго-пластических течений // Вычислительные методы в гидродинамике. 1967. М. Изд-во: Мир. С. 212-263. 